Arthritis in a Specialized Care Clinic. Reumatología Clínica (English Edition). 2016;12(6):313-8.

[2] Purabdollah M, Lakdizaji S, Rahmani A, Hajalilu M, Ansarin K. Relationship between Sleep Disorders, Pain and Quality of Life in Patients with Rheumatoid Arthritis. Journal of caring sciences. 2015;4(3):233-41.

Disclosure of Interest: None declared

DOI: 10.1136/annrheumdis-2017-eular.5639

\section{OP0258-HPR INTENSIVE PHYSICAL EXERCISE FOR ELDERLY PERSONS WITH RHEUMATOID ARTHRITIS IMPROVES PHYSICAL CAPACITY}

E. Lange ${ }^{1,2}$, D. Kucharski ${ }^{3}$, K. Svensson ${ }^{4}$, S. Svedlund ${ }^{3}$, I. Gjertsson ${ }^{2,3}$, K. Mannerkorpi ${ }^{1,2}$. ${ }^{1}$ Department of Health and Rehabilitation, Institute of neuroscience and physiology, Sahlgrenska academy, University of Gothenburg; ${ }^{2}$ University of Gothenburg Centre for Person-centred Care; ${ }^{3}$ Rheumatology and inflammation research, Institute of medicine, Sahlgrenska academy, Göteborg;

${ }^{4}$ Skaraborg hospital, Skövde, Sweden

Background: Today, more than $50 \%$ of persons with Rheumatoid Arthritis (RA) are over 65 years of age (1). Little is known about the effects of physical exercise in this age group ( $>65$ years).

Objectives: The aim of this randomized controlled study is to investigate the effects of a person-centred progressive aerobic and resistance exercise program, led by a physiotherapist.

Methods: Seventy-four with persons with RA ( $24 \%$ men), mean age 70 years (SD 2.5), were recruited and randomized to an exercise interventions group or an active control group. The intervention consisted of a 20-week individual person-centered exercise program, performed three times a week with guidance from a physiotherapist. Both aerobic and resistance exercise was performed on a high intensity level. The control group followed a home exercise program twice a week. Muscle strength and endurance were assessed by the Chair Stands test, the Timed up and Go and a Bicycle endurance test. Maximal aerobic capacity $\left(\mathrm{VO}_{2} \mathrm{max}\right)$ was assessed with ergo spirometry. Activity limitations were assessed by SF36 Physical subscale and the Health Assessment Questionnaire (HAQ)

Results: All participants in the intervention group completed the intervention. The participants had a low disease activity with a mean Clinical Disease Activity Index of 5.4 (SD 3.9). Significant improvements were found for $\mathrm{VO}_{2}$ max, the Chair Stands test, the Timed up and Go, the Bicycle endurance test on bicycle $(p<0,001)$ and the SF36 physical $(p=0.018)$ in the intervention group, when compared to the controls. No significant differences between groups were seen on $\mathrm{HAQ}$.

Conclusions: Intensive progressive aerobic and resistance exercise is a feasible intervention for elderly persons with RA. Despite old age and RA the participants gained significant improvements in physical capacity.

\section{References:}

[1] Eriksson JK, Neovius M, Ernestam S, Lindblad S, Simard JF, Askling J. Incidence of rheumatoid arthritis in Sweden: a nationwide population-based assessment of incidence, its determinants, and treatment penetration. Arthritis Care Res (Hoboken). 2013;65(6):870-8.

Disclosure of Interest: None declared

DOI: 10.1136/annrheumdis-2017-eular.2721

\section{OP0259-HPR SUPERVISED WALKING IMPROVES AEROBIC CAPACITY EXERCISE TOLERANCE, FATIGUE AND PERCEIVED IMPROVEMENT IN WOMEN WITH PRIMARY SJÖGREN'S SYNDROME: A RANDOMIZED CONTROLLED TRIAL}

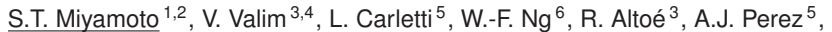
L.H. Dias ${ }^{3}$, É.V. Serrano ${ }^{3}$, A.M. Subtil ${ }^{5}$, V.B. Cândido ${ }^{5}$, M. Trenell ${ }^{7}$ D. Lendrem ${ }^{6}$ J. Natour ${ }^{2}{ }^{1}$ Departamento de Educação Integrada em Saúde, Universidade Federal do Espírito Santo, Vitória; ${ }^{2}$ Disciplina de Reumatologia, Universidade Federal de São Paulo, São Paulo; ${ }^{3}$ Serviço de Reumatologia do HUCAM; ${ }^{4}$ Departamento de Clínica Médica; ${ }^{5}$ Laboratório de Fisiologia do Exercício (LAFEX), Universidade Federal do Espírito Santo, Vitória, Brazil; ${ }^{6}$ Musculoskeletal Research Group, Institute of Cellular Medicine; ${ }^{7}$ MoveLab, Physical Activity \& Exercise Research, Institute of Cellular Medicine, Newcastle University, Newcastle upon Tyne, United Kingdom

Background: Fatigue is a very common symptom of primary Sjögren's syndrome (pSS), being reported by up to $70 \%$ of patients [1]. It is more pronounced when compared to healthy individuals [2] and patients often report that it is their greatest problem and the most difficult to cope with [3]. There is only one non-randomized controlled study on aerobic exercise in pSS with a small sample size suggesting improvement in fatigue, aerobic capacity, depression and physical function [4]. Objectives: To evaluate the safety and effectiveness of a supervised walking program in women with pSS.

Methods: Forty five sedentary women fulfilling the American European Consensus Criteria for pSS were randomized to a Training Group (TG, $n=23$ ) or Control Group ( $C G, n=22$ ). Patients in the $T G$ were submitted to supervised walking, 3 times a week, for 16 weeks. The patients of the $C G$ were instructed to not perform any kind of regular physical exercise. Outcomes measured were aerobic capacity, fatigue, disease activity, depression, perception of pSS's symptoms and quality of life. An intent-to-treat analysis was performed.

Results: The mean change after 16 weeks of $\mathrm{VO}_{2 \max }\left(\mathrm{ml} \cdot \mathrm{kg}^{-1} \cdot \mathrm{min}^{-1}\right)$, distance and FACIT-fatigue were higher in the TG than in the CG $(p=0.016, p=0.043$ and $p=0.030$, respectively) (Figure 1 ). Improved aerobic capacity was associated with improvements in fatigue scores and physical components of quality of life measured using SF-36. Furthermore, improved fatigue scores were associated with reduced depression and improvements in the physical and mental components of the quality of life measures. Overall, $95.4 \%$ of patients in the TG rated themselves as clinically improved versus $62 \%$ of the patients in the $C G(p=0.049)$. There was no flare in disease activity and no serious adverse events with exercise.
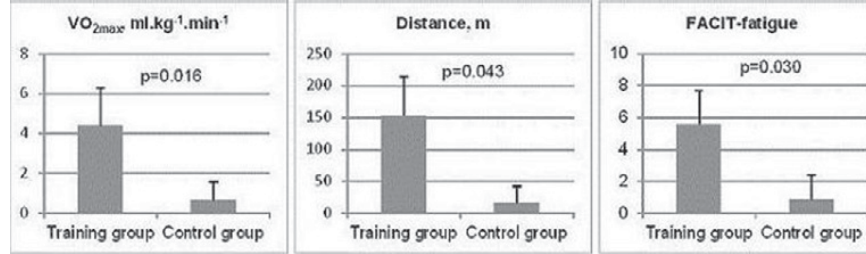

Figure 1. The exercise training group showed improved aerobic capacity, improved exercise tolerance and improved fatigue compared to controls.

Conclusions: This supervised walking program was demonstrated to be feasible and safe with improvements in the aerobic capacity, exercise tolerance, fatigue and patient perception of improvement in pSS patients.

References:

[1] Ng W-F, Bowman SJ. Primary Sjogren's syndrome: too dry and too tired. Rheumatology 2010;49(5):844-53.

[2] Strömbeck B, Ekdahl C, Manthorpe R, et al. Physical capacity in women with primary Sjögren's syndrome: a controlled study. Arthritis Rheum 2003:49(5):681-8.

[3] Meijer JM, Meiners PM, Huddleston Slater JJR, et al. Health-related quality of life, employment and disability in patients with Sjogren's syndrome. Rheumatology 2009;48(9):1077-82.

[4] Strömbeck BE, Theander E, Jacobsson LTH. Effects of exercise on aerobic capacity and fatigue in women with primary Sjogren's syndrome. Rheumatology 2007;46(5):868-71.

Acknowledgements: This work was supported by the Coordenação de Aperfeiçoamento de Pessoal de Nível Superior - CAPES Foundation [BEX 8831/14-9 to S.T.M].

Disclosure of Interest: None declared

DOI: 10.1136/annrheumdis-2017-eular.3854

\section{OP0260-HPR HIGHER SATISFACTION WITH ACTIVITY-RELATED SYMPTOMS AFTER 15-WEEK RESISTANCE EXERCISE IN WOMEN WITH FIBROMYALGIA}

K. Mannerkorpi ${ }^{1,2}$, A. Larsson ${ }^{2}$, A. Palstam ${ }^{1}$, M. Ernberg $^{3}$, B. Gerdle ${ }^{4}$, E. Kosek ${ }^{5} .{ }^{1}$ Inst of Neuroscience and physiology; ${ }^{2}$ Gothenburg Center of Person-centred Care (GPCC), University of Gothenburg, Göteborg; ${ }^{3}$ Department of Dental Medicine, Karolinska Institute, Stockholm; ${ }^{4}$ Department of Medical and Health Sciences, Linköping University, Linköping; ${ }^{5}$ Department of Clinical Neuroscience, Karolinska Institute, Stockholm, Sweden

Background: Physical exercise is troublesome for most patients with fibromyalgia (FM) due to activity-induced pain. A reason for activity-induced pain is a low pain threshold. In the present study we investigated if experience of physical activity changed after 15-week progressive person-centred resistance exercise. The control group participated in 15-week relaxation program.

Objectives: To investigate how experience of physical activity changed after 15 -week resistance exercise in women with FM, and if experiences correlated with pain threshold.

Methods: 130 women (age 22-64 years, symptom duration 0-35 years) with FM were randomized to 15 -week resistance exercise or to a parallel relaxation program. The participants completed Experience of physical activity scale (EPA) comprising five subscales (0-7), assessing how exercise was perceived in terms of Physical relaxation (PR), Well-being (WB), Activity beliefs ( $A B$ ), Activity-related symptoms (ARS), and Activity Habits (AH) (1). A lower score indicates a higher satisfaction. Pain threshold was investigated with algometer. Within-group and between-group analyses were conducted by non-parametric statistics. Correlations between algometry and ratings on EPA were investigated by Spearman correlation coefficient.

Results:

The resistance exercise group scored significantly higher satisfaction at posttest than before the intervention in their ratings on how they experienced exercise in terms of PR, WB, ARS and AH $(p<0.05)$, Table 1.

Between-group analyses showed that the resistance exercise group scored significantly higher satisfaction in ARS subscale $(p<0.006)$ after the intervention when compared to the relaxation group.

Significant correlations were found between algometry and PR ( $r s-0.32, p=0.017$ ) as well as ARS $(-0.33, p=0.015)$ at post-test in the resistance exercise group. 